\title{
Los Sistemas de Información Geográfica y el registro arqueológico del Chaco Meridional
}

\author{
Geographical Information Systems and Archaeological Record in Southern \\ Chaco
}

\author{
Guillermo Lamenza ${ }^{1}$ Catalina Mostaccio ${ }^{2}$ Maria de los Ángeles Andolfo ${ }^{1}$ Horacio Calandra ${ }^{3}$ \\ ${ }^{1}$ Becario. FCNyM-UNLP. ${ }^{2}$ Prof. Adj. Fac. de Informática-UNLP. CICPBA ${ }^{3}$ Investigador.IIGH CONICET \\ glamenza@fcnym.unlp.edu.ar
}

\section{Resumen}

El presente trabajo forma parte de un proyecto en ejecución denominado "De las Historias Étnicas a la Prehistoria en el Gran Chaco" donde concurren varias disciplinas antropológicas y otras afines, tendiendo al estudio integral y sistemático de la historia desde los grupos sociales vivientes hacia el pasado, partiendo del punto de vista interno de esas sociedades para colaborar en la recuperación de sus historias étnicas.

En el caso particular de la arqueología, han sido muchos los avances logrados en el último decenio, gracias a los aportes de diferentes grupos de investigación, lo que nos permite dar cuenta de la importancia del registro arqueológico regional y comenzar a esbozar los distintos aspectos caracterizantes que le dan identidad propia a la región. En este estado de avance, los Sistemas de Información Geográfica (SIG) constituyen una herramienta metodológica de gran importancia para la arqueología, teniendo en cuenta que su aplicación ha estado vinculada a estudios en diferentes escalas espaciales y a distintas resoluciones temporales, siendo de utilidad tanto en el proceso de investigación como en la gestión y difusión del patrimonio arqueológico.

En esta oportunidad destacaremos la importancia de la incorporación de los SIG al estudio del registro arqueológico regional chaqueño y presentaremos el Mapa Temático Arqueológico con la localización de más de 100 sitios, su sectorización y la elaboración de un sistema unificado de descripción que permita facilitar la comparación y que sea de utilidad para futuras investigaciones. También nos interesa poner en discusión algunos problemas surgidos en lo referente al acceso y actualización de esta información mediante la implementación de aplicaciones Web.

\section{Abstract}

This paper is part of a project under implementation called "De las Historias Étnicas a la Prehistoria en el Gran Chaco" (From Ethnic History to Prehistory in Gran Chaco) where various anthropologic and other similar disciplines combine tending to the comprehensive and systematic study of history from living social groups to the past. The study takes the inner point of view of those societies as the starting point to cooperate in recovering their ethnic histories.

The progress made in the past decade in Archaeology has been significant thanks to the contributions of different research groups. This allows us to explain the importance of the regional 
archaeological record and to outline the different characterizing aspects that give the region its own identity. In this state of affairs, Geographical Information Systems (GIS) constitute a very significant methodological tool for archaeology, considering that their application has been linked to studies in different spatial scales and temporal resolutions, being useful both in the research process and in managing and disseminating the archaeological patrimony.

We will highlight the importance of incorporating GIS to the study of the regional archaeological record of el Chaco and present the Archaeological Theme Map with the localization of more than 100 sites, their sectorization and the creation of an unified description system which enables comparison and could be used in future researches. We would also like to discuss some problems regarding the access and updating of this information using Web applications.

\section{Introducción}

El presente trabajo forma parte de un proyecto en ejecución al que concurren varias disciplinas antropológicas y otras afines, tendiendo al estudio del Gran Chaco Meridional. Este proyecto, denominado "De las Historias Étnicas a la Prehistoria en el Gran Chaco" propone una investigación comparativa, regional y multidisciplinaria, haciendo historia desde los grupos sociales vivientes hacia el pasado, partiendo del punto de vista interno de esas sociedades para colaborar en la recuperación de sus historias étnicas (Braunstein et al, 2002).

En lo referente al campo de la arqueología, la historia de las investigaciones en la región se ha caracterizado por valiosas comunicaciones, aunque discontinuas y esporádicas, que pueden remontarse a comienzos del siglo XX de la mano de la misión sueca "Chaco-cordillera" (Nordenskiöld, 1903; von Rosen, 1957).

Hasta hace no mucho tiempo atrás, se mencionaba al registro arqueológico chaqueño por extensión y visto desde la periferia, relacionado con la Región de las Selvas Occidentales, la Región Chaco-Santiagueña y la Subárea del Nordeste argentino, siendo casi una constante hacer referencia a su desarrollo cultural en vinculación con el Área Andina y/o Amazónica. Esta situación ha hecho lícito que se defina al Chaco Meridional como el "Agujero Negro" de la arqueología sudamericana (Braunstein et al, op cit). En la última década, gracias a los aportes de diferentes grupos de investigación, se ha comenzado a revertir esta situación y el registro arqueológico regional ha ido adquiriendo identidad propia.

Dadas las características propias del área -tales como la escasa información relativa a su extensión territorial y por lo tanto con evidencias culturales parciales- los lugares de asentamiento y la alfarería, constituyeron los indicadores fundamentales para reconstruir el proceso cultural general (Dougherty y Zagaglia, 1982; Calandra y Dougherty, 1991; Dougherty et al., 1992).

Con esta premisa se desarrollaron los primeros trabajos, abarcando el territorio en forma extensiva, antes que intensiva y localizada, lo cual nos mostró un espectro cultural multivariado correlacionable con los aspectos ambientales (De Feo et al., 2002). El incremento de los trabajos de campo y el análisis de la información obtenida permitieron un primer ordenamiento del área en tres sectores ecológico-culturales (Salceda y Calandra, 2003; Calandra y Salceda, 2004) que se interrelacionan a través de los cursos de agua principales o secundarios, activos o no en la actualidad. (fig. 1). 

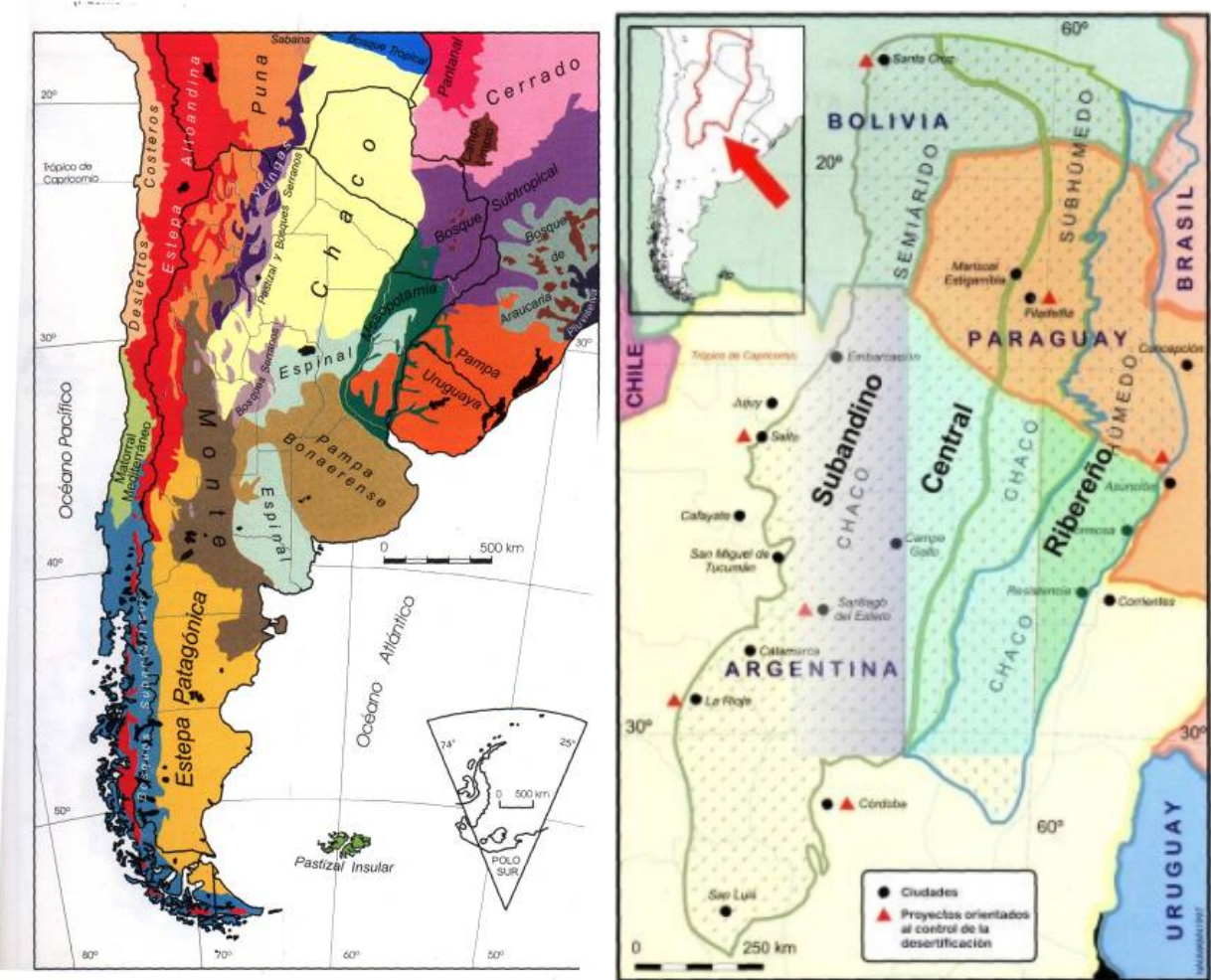

Figura 1. Mapa del Gran Chaco con sectorización Ecológico-Cultural (modificado de Naumann, 1999).

Las investigaciones intensivas, realizadas hasta el momento sólo en algunos asentamientos, en particular los ubicados en el extremo norte del Sector Central y en el Ribereño Paraguay-paranaense, nos llevan a una etapa de la investigación que incluye la determinación de cambios desde una óptica temporal y un análisis ecológico más detallado, facilitando de este modo el planteo comparativo etnográfico-paleoetnográfico.

Los trabajos arqueológicos desarrollados en la región incluyen análisis de la ergología procedente de los sitios investigados (Calandra et al., 2000; Calandra et al., 2001; Calandra et al., 2003; Balbarrey et al., 2003; Calandra et al, 2004; Lamenza et al, 2005), de las relaciones con áreas vecinas (Calandra et al., 2003; Sempé et al., 2003; Calandra y Salceda, op cit), de los materiales faunísticos asociados (Salceda et al., 2000; Santini et al., 2003), de los modos de inhumación y restos óseos humanos involucrados (Colazo et al., 2001; Méndez et al., 2003; Colazo et al., 2004; Desántolo et al., 2005), así como trabajos de síntesis (Salceda y Méndez, 1999; Méndez et al., 2000; Tomasini et al., 2003; Calandra et al., 2002; Salceda y Calandra, op cit; Calandra y Salceda, op cit). También es importante destacar que en los últimos años se retomaron las investigaciones en el sitio arqueológico Km. 75, bajo la dirección de la Lic. Susana Colazo (Colazo y Guarino, 2005).

A partir de los datos éditos e inéditos, en concordancia con normas establecidas y explícitas convenidas en reuniones de trabajo entre especialistas (Primera Convención Nacional de Antropología-Segunda Parte, 1965; Tarrago y Núñez Regueiro, 1972; Calandra y Dougherty, 198485), se localizaron y caracterizaron culturalmente todos los sitios arqueológicos registrados hasta el año 2001(De Feo et al, 2002). Como concluyéramos en esa oportunidad, la elaboración de un mapa arqueológico requiere de constantes aportes y actualizaciones. Atender a esta problemática resulta de suma importancia como instancia inicial para la puesta en valor, gestión y difusión del patrimonio arqueológico regional.

La aplicación de los SIG a la problemática arqueológica. 
Ante la constante búsqueda de recursos metodológicos que, mediante su incorporación efectiva, contribuyan a clarificar el conocimiento sobre los desarrollos de las sociedades pasadas en la región, consideramos a los Sistemas de Información Geográfica (SIG) como una herramienta fundamental, cuyo potencial dista de ser actualmente aprovechado en su totalidad.

Provenientes de campos como la Informática y la Geografía, no hay que dejar de tener en cuenta que los SIG son sistemas informáticos que solo nos serán de utilidad, mientras se defina un marco teórico y se utilicen para resolver problemas arqueológicos concretos (Barceló y Pallares, 1996). Aunque las primeras aplicaciones estuvieron vinculadas con los lineamientos teóricos de la arqueología espacial, hoy puede verse una vasta diversidad temática y conceptual en torno a su utilización, lo que permite minimizar los riesgos de la simulación informática y de una percepción exclusivamente determinista de las causas que rigen los patrones de asentamiento humanos.

La utilización de los SIG ha aumentado notablemente en los últimos 10 años. En nuestro país, día a día crece el número de colegas que implementan SIG en sus áreas y temáticas. De manera ilustrativa, en el marco del XV Congreso Nacional de Arqueología Argentina, se generó un simposio específico para atender a la problemática del uso de los SIG en la Arqueología (Libro de Resúmenes del XV Congreso Nacional de Arqueología Argentina, 2004) donde quedó plasmada la flexibilidad de la herramienta y la creatividad para su aplicación a los distintos problemas. La mayoría de las aplicaciones se refieren a una primera etapa de trabajo, donde los SIG son vistos como el entorno que permite referenciar geográficamente la información gráfica obtenida y relevada en los trabajos de campo, y combinarla con los datos alfanuméricos que dan cuenta de los distintos análisis efectuados sobre los sitios y los materiales presentes en ellos, organizados en planillas o bases de datos.

En la región que nos ocupa, una de las cuestiones principales era establecer y asentar una importante cantidad de sitios, algunos identificados espacialmente por los autores, y otros no; trabajando principalmente a una escala que podríamos denominar regional. Precisamente, una de las potencialidades de esta herramienta es la facilidad con que se pueden abordar las distintas escalas, pasando desde el nivel de sitio o localidad arqueológica, a una dimensión mayor, regional, donde se identifican distintos sectores ecológico-culturales.

Otra cuestión de importancia era la unificación de toda la información existente en la zona, en una base de datos general, y su previa corrección, homogenización y/o codificación, con miras a su posterior utilización en la construcción de mapas temáticos. En este sentido, es también muy relevante la ayuda de los sistemas en relación a la presentación de los resultados obtenidos por las investigaciones, y la posibilidad de una actualización eficiente.

Vista la importancia de la utilización de los SIG en el campo de la arqueología y respondiendo al problema concreto, presentamos los resultados de la incorporación de la información en un entorno SIG, conformando un Mapa Temático Arqueológico, y la propuesta de implementación de una aplicación Web para su constante actualización, gestión y fácil acceso para todos los interesados en la información referente al registro arqueológico regional.

\section{a) Mapa Temático Arqueológico}

La unidad principal para la confección de este mapa fue el Sitio Arqueológico. Según una definición comúnmente aceptada, un sitio arqueológico es un lugar donde pueden hallarse huellas de acción humana (Renfrew y Bahn, 2000). Siguiendo los lineamientos metodológicos expuestos en De Feo et al. (2002) y continuando con el ordenamiento que comenzara en la provincia de Formosa (Calandra y Dougherty, op cit; Dougherty et al., op cit) se construyó una base de datos con los sitios registrados hasta el 2001 (incluyendo las provincias de Chaco y Salta) y se actualizó con la información édita e inédita disponible hasta la fecha de esta presentación (Calandra et al, 2004; notas de campo Lic. Tobisch; notas de campo Lic Calandra; notas de campo Lic. Santini-Lamenza). Las referencias espaciales fueron unificadas, en los casos en que esto fue posible, a coordenadas geográficas en grados decimales. Los datos fueron incorporados a un SIG (ArcView GIS 3.2). 
Como esbozáramos anteriormente, no se pudo identificar espacialmente la totalidad de los sitios que conforman el Mapa Arqueológico. De un total de 109 sitios registrados, el 57\% no tenía referencias espaciales absolutas (fig. 2). Esto presentaba una dificultad, ya que los sitios referidos sólo por la cercanía con algún centro poblado conocido, no podrían ser ingresados en el sistema de la misma forma que los sitios con referencia espacial absoluta. En consecuencia, se generaron dos capas temáticas, una incluye a los sitios identificados y la otra a los sitios sin referencia espacial absoluta. Los sitios identificados se representan con puntos y las zonas de probable ubicación de los sitios sin referencia se representan con polígonos. Cada polígono está asociado a una tabla que contiene un subconjunto de dichos sitios. (fig 3). Con esta información se creo el Mapa Temático Arqueológico (fig 4)

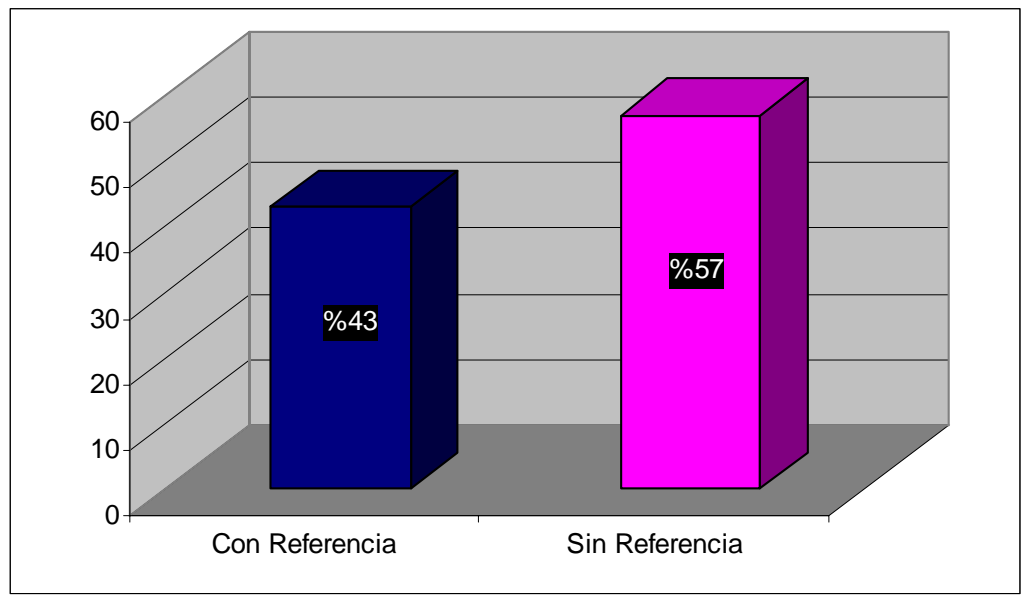

Figura 2. Distribución porcentual de sitios con/sin referencia espacial absoluta

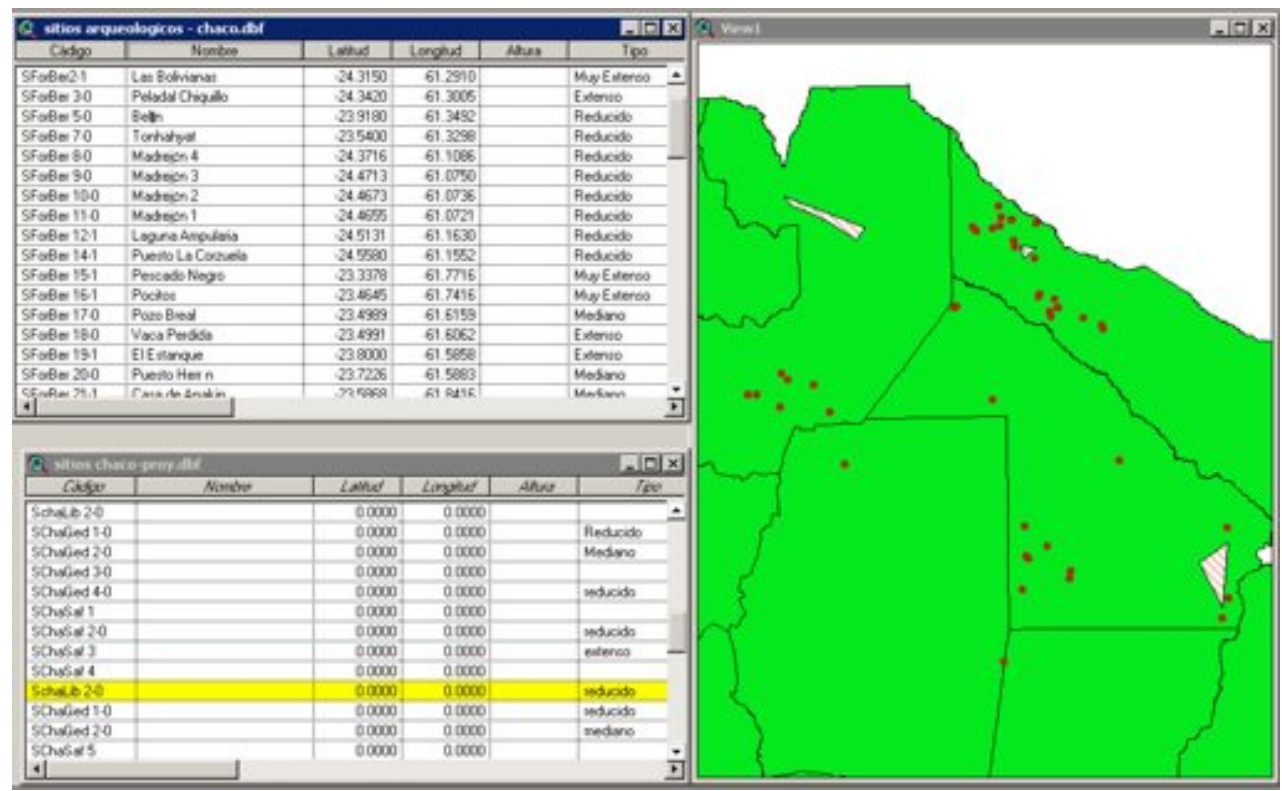

Figura 3. Tablas de los sitios con/sin referencia. 


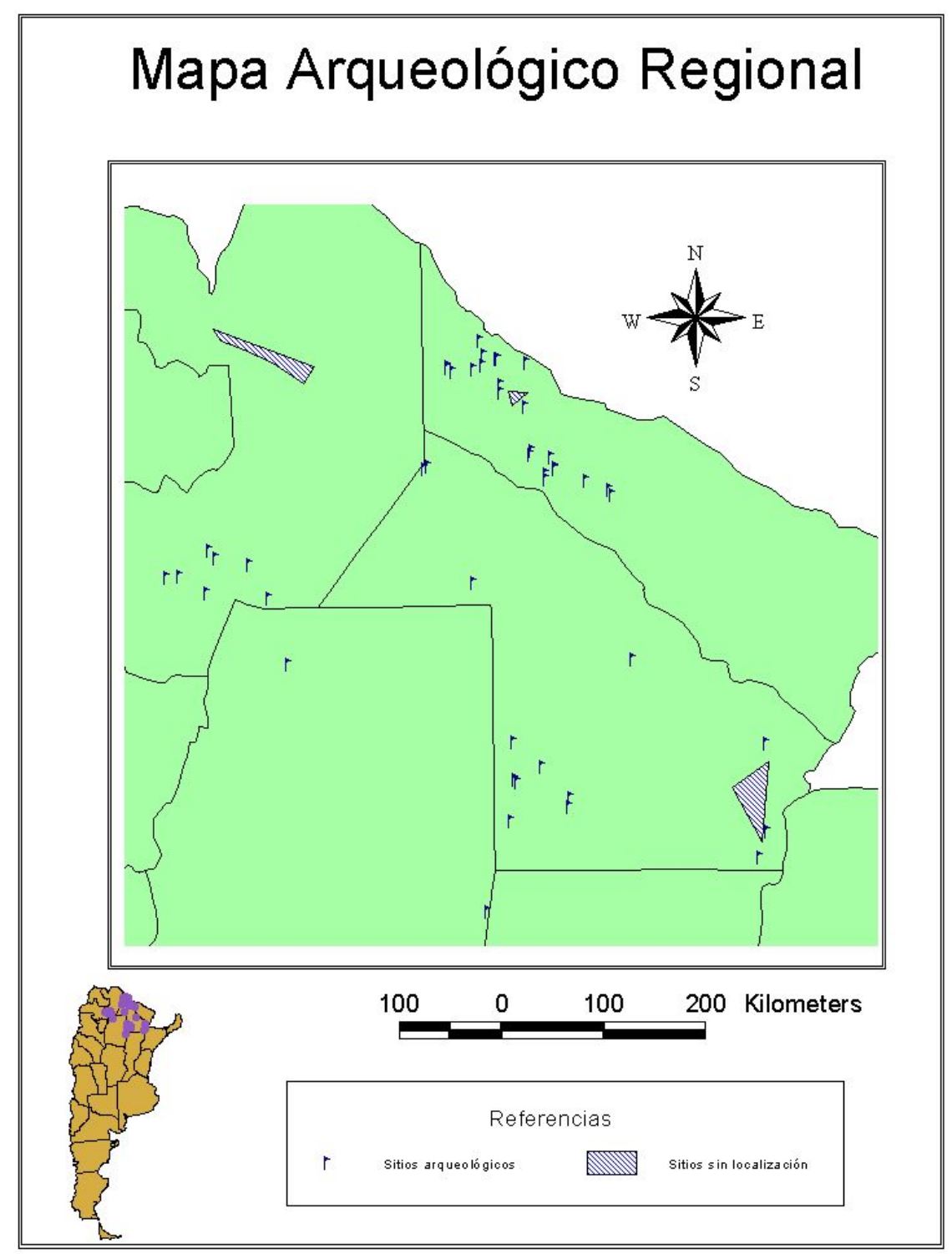

Figura 4. Mapa Temático Arqueológico

\section{b) De los sitios arqueológicos al sitio web}

La World Wide Web o simplemente Web fue creada por Tim Berners-Lee (http://www.w3.org/People/Berners-Lee/) en 1989, en aquel momento Internet era utilizado casi exclusivamente por un grupo reducido de investigadores para intercambiar documentos científicos. Inicialmente, la Web ofrecía sólo acceso a documentos estáticos; sin embargo, la constante evolución de las tecnologías en que se apoya, la han convertido en un potente y versátil medio de comunicación.

En esta sección describiremos la aplicación Web propuesta para abordar la visualización y consulta del Mapa Temático Arqueológico, y la actualización del mismo.

La visualización y consulta del Mapa en la Web se obtiene usando el ambiente de desarrollo MapServer ( http://ms.gis.umn.edu/ ) para construir, a partir de las capas temáticas y la base de datos descriptas en la sección anterior, un sitio Web. El usuario accederá a él a través de su Browser local haciendo la invocación a la URL correspondiente. (fig 5) 


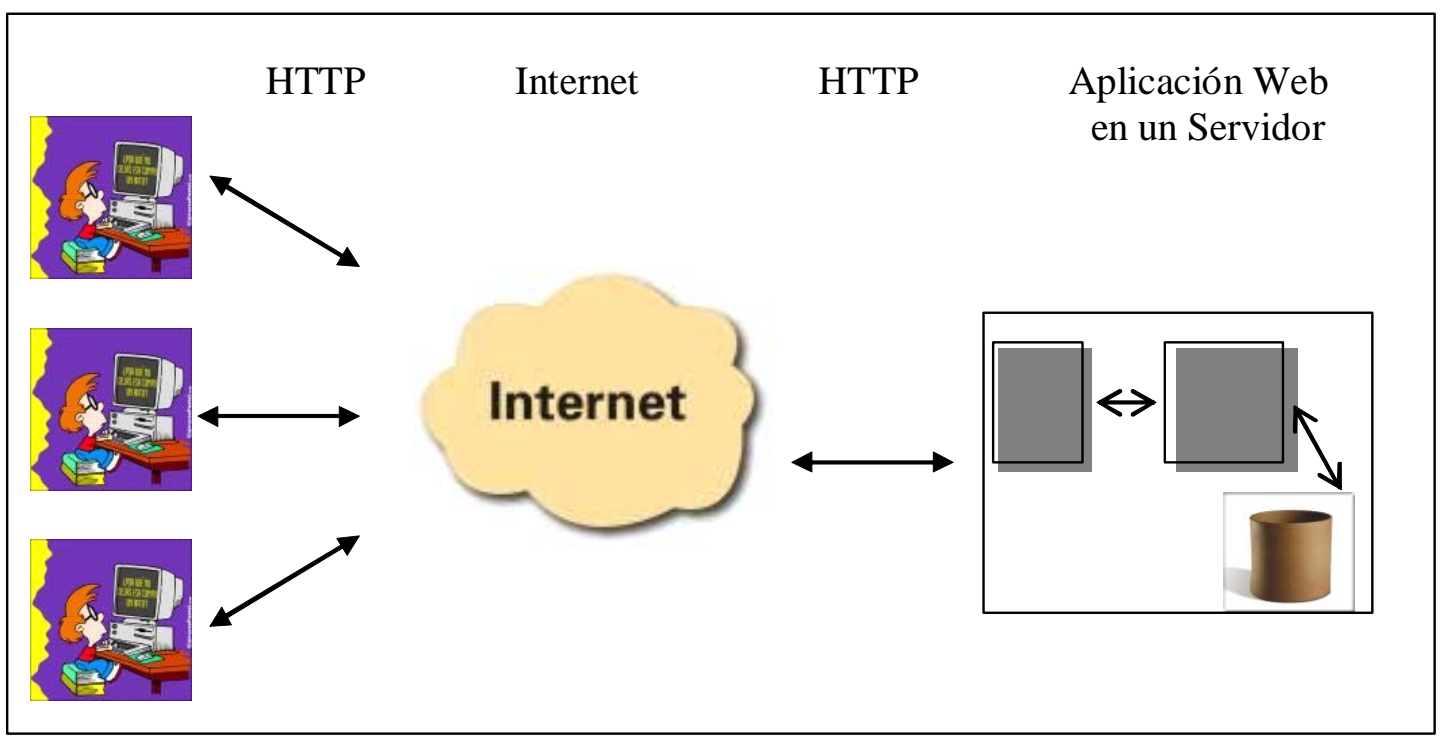

Figura 5. Esquema general de comunicación entre el usuario y la aplicación

Para mantener actualizada la base de datos, esta aplicación permitirá:

- ingresar nuevos sitios

- modificar información de sitios existentes

- manifestar observaciones sobre información de sitios existentes

A continuación mencionamos algunas de las consideraciones tenidas en cuenta al momento de definir la aplicación.

\section{Seguridad de acceso}

Uno de los objetivos principales de esta aplicación es proveer datos lo más actualizados posible a los investigadores interesados en la Región. Para el logro de este objetivo, se deben dar las herramientas tanto a quienes se interesen en registrar nuevos sitios, como a quienes modifiquen información de los sitios ya existentes, para que puedan actualizar el Mapa. Como consecuencia inmediata de lo anterior, se deben tomar todos los recaudos a fin mantener la integridad de la base de datos subyacente. Hemos incorporado un nivel de seguridad, que implica restringir el acceso a los datos, definiendo Perfiles para los usuarios autorizados. La administración de los permisos asignados a cada nivel se realiza mediante un módulo especialmente diseñado para esa tarea.

En la Tabla 1 se muestran los perfiles definidos con el nivel de acceso permitido. Los permisos asignados a cada uno son los siguientes:

- el Visitante sólo puede visualizar el mapa.

- el Miembro Visitante está sólo autorizado a registrar Observaciones.

- el Miembro Autor está autorizado a Ingresar Nuevos Sitios y/o Modificar Sitios de su Autoría e incluye las autorizaciones del Miembro Visitante. Para obtener este perfil de usuario, se deberá presentar una acreditación diferente de la requerida para el ingreso como Miembro Visitante.

- el Administrador tiene acceso al 100\% de los sitios e incluye las autorizaciones de los perfiles anteriores. 
Perfil del usuario

\begin{tabular}{|c|c|}
\hline Administrador & $\begin{array}{lr}\text { Lectura: } \quad 100 \% \\
\text { Escritura: } \quad 100 \% \\
\text { Visualización: } & 100 \%\end{array}$ \\
\hline Miembro Autor & $\begin{array}{ll}\text { Lectura: } & 100 \% \\
\text { Escritura: } & \text { sólo a los de su } \\
\text { autoría } \\
\text { Visualización: } 100 \%\end{array}$ \\
\hline Miembro Visitante & $\begin{array}{cr}\text { Lectura: } & 100 \% \\
\text { Escritura: } & \text { sólo } \\
\text { comentarios } & \\
\text { Visualización: } 100 \%\end{array}$ \\
\hline Visitante & $\begin{array}{lc}\text { Lectura: } & 100 \% \\
\text { Escritura: } & 0 \% \\
\text { Visualización: } & 100 \%\end{array}$ \\
\hline
\end{tabular}

Tabla 1: Perfil del usuario y nivel de acceso a los datos

La información que deseamos mantener almacenada y actualizada de cada sitio arqueológico, y que conformaría los campos de cada registro es la siguiente:

Identificación codificada, Otros nombres, Ubicación, Tipo de yacimiento, Descripción general, Bibliografía, Documentación inédita, Tipo de relevamiento

Agregamos a ella un campo Autor, identificando al Investigador que ingresó los datos del sitio, el cual será el único usuario autorizado por la aplicación a realizar modificaciones sobre dicho sitio.

\section{Funcionalidades de la aplicación}

A continuación, introduciremos las funciones de la aplicación a través de la descripción de los escenarios posibles para los usuarios.

Escenario 1: un usuario desea registrarse como miembro del sitio.

1.1. Se le pedirá completar una planilla con diferentes grados de exigencia según el perfil solicitado.

1.2. Una vez aprobada la solicitud se le envía un mail con su contraseña.

Escenario 2: un usuario encontró un nuevo sitio arqueológico y desea publicarlo en el sitio Web.

2.1. Se le pedirá su nombre de usuario y contraseña. Se pueden presentar dos situaciones:

2.1.a. es miembro del sitio Web: se validará la información del usuario como "Miembro Autor"

2.1.b. no es miembro del sitio Web: se solicitará que se registre ingresando sus datos, para luego validarlo como "Miembro Autor" 
2.2. Se desplegará el siguiente formulario para completar los datos del sitio:

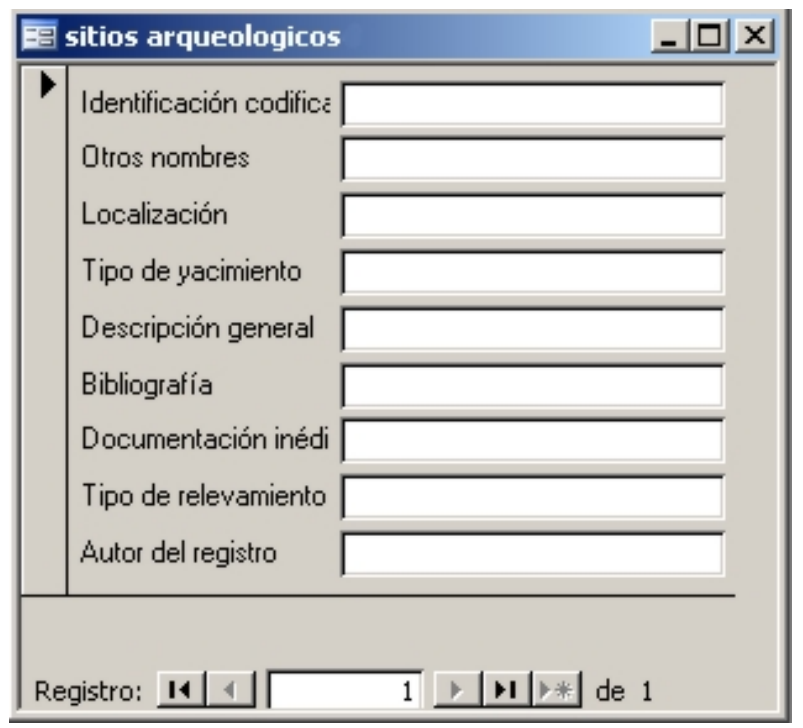

Escenario 3: un usuario desea realizar modificaciones a un sitio.

3.1. Idem 2.1

3.2. Se le pedirá la "Identificación Codificada" (Ej. SForMat 1-1) del sitio a modificar y se validará que el usuario sea Autor de dicho sitio.

3.3. Se desplegarán los datos del sitio solicitado para que el Autor realice las modificaciones.

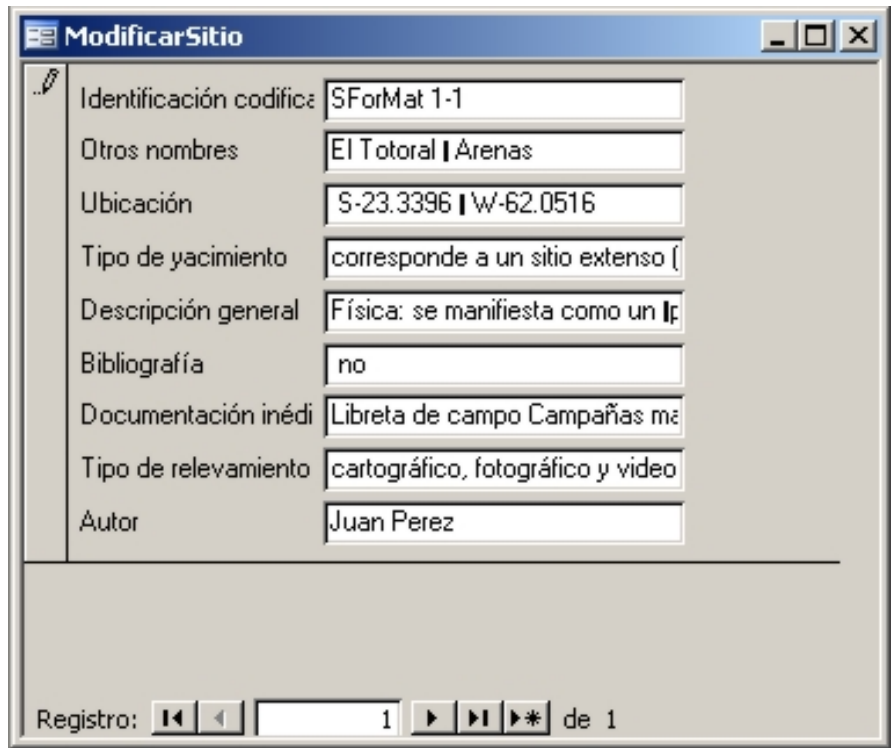

Escenario 4: un usuario que visita el sitio Web, desea asentar algunas observaciones a la información presentada, por ejemplo, agregar y/o corregir algún dato descriptivo; y que estas observaciones sean conocidas por futuros usuarios. 
4.1. Idem 2.1, pero indicándole que si no es miembro debe registrarse. (Solo los miembros visitante y los niveles superiores podrán ingresar comentarios)

4.2. Se validará si es miembro del sitio

4.3. Se desplegará un panel para que escriba sus Observaciones.

Las funciones definidas previamente responden a las necesidades expresadas por los investigadores que utilizarán la aplicación. La respuesta de la aplicación en cada uno de los escenarios es un diseño preliminar de cada función. La próxima etapa será la implementación, previa evaluación y elección de las tecnologías a utilizar.

\section{Consideraciones Finales}

En esta presentación hemos pretendido mostrar los primeros pasos en la utilización de los SIG para la resolución de un problema arqueológico concreto.

Un mapa arqueológico tradicional, impreso en papel, presenta una limitación en cuanto a su situación estática, que implica una rápida desactualización y el manejo de la información a una escala espacial fija. Los SIG, por el contrario, posibilitan la rápida visualización de la información, el manejo de distintas escalas de análisis, y combinar información de distinta naturaleza que, junto a la implementación de la aplicación web propuesta, permiten la actualización constante y accesible para los distintos grupos de investigación interesados.

Teniendo en cuenta el gran potencial de los SIG aplicados a la arqueología, queremos resaltar la importancia de su utilización en los distintos niveles de análisis, tanto en la investigación científica como en la gestión del patrimonio y, ligados a otras tecnologías (en este caso, aplicaciones web), en la divulgación de la información arqueológica (Espiago y Baena, 1999).

Específicamente siendo en la actualidad la identificación y preservación del patrimonio cultural un aspecto preponderante, no nos cabe duda que mediante esta propuesta, facilitamos enormemente tal gestión y proponemos base sólida para su implementación en relación de dependencia con entes nacionales y/o provinciales en los cuales recaiga esta responsabilidad.

\section{Referencias citadas}

XV Congreso Nacional de Arqueología Argentina. 2004. Libro de resúmenes y programa General de Actividades. Pp. 183-196.

Balbarrey G., Calandra H.A., Couso G., Lamenza G., Aguirre B., 2003. Nuevos aportes al análisis cerámico del sector central del Gran Chaco argentino. En Actas del XXIII Encuentro de Geohistoria Regional. Pp. 350-363.

Barceló J.A., PALLARES M., 1996 A critique of GIS in Archaeology. From Visual seduction to Spatial Analysis. Archeologia e Calcolatori No. 6.

Braunstein J.A., S.A. Salceda, H.A. Calandra, M.G. Méndez, S.O. Ferrarini.. 2002, Historia de los chaqueños - Buscando en la "papelera de reciclaje" de la antropología sudamericana. Acta Americana, Journal of de Swedish Americanist Society. Vol. 10 (1): 59-88. Suecia.

Calandra H. y B. Dougherty B. 1991. Prospección arqueológica en la provincia de Formosa, República Argentina. En: Hacia una Nueva Carta Étnica del Gran Chaco pp 133-141. Centro del Hombre Antiguo Chaqueño (CHACO). Las Lomitas, Pcia. de Formosa, Argentina.

Calandra H.A., Méndez M.G., Salceda S.A., Lamenza G., Lanciotti M., Del Papa L., Duhalde N. 2000. Estudio preliminar de los restos cerámicos hallados en el sitio arqueológico "El Cachapé" (Chaco, Argentina). En Actas del XX Encuentro de Geohistoria Regional. Vol I: 157-171.

Calandra H.A., Ferrarini S.O., Santini M., 2001. Alfarería vinculante entre Selvas Occidentales y Chaco Meridional. Milenio. Pp 117-119. 
Calandra H.A., Ferrarini S.O., Méndez M.G., Salceda S.A., Iglesias T., Lema V., 2002. Potencialidad sustentadora de la región meridional del Gran Chaco. Arqueología Histórica Argentina. Actas del Primer Congreso Nacional de Arqueología Histórica. Pp: 435-445. Ed. Corregidor, Mendoza.

Calandra H.A., Ferrarini, S.O., 2003. Arqueología Chaqueña 1. Antecedentes. En Actas del XIII Congreso Nacional de Arqueología Argentina. Universidad Nacional de Córdoba. Ed. Brujas. Tomo 3: 65-72.

Calandra H.A, Salceda S.A., González O., Cid de la Paz M., Caló M., 2003. Arqueología Chaqueña 3: nuevas evidencias de cordelería impresa. En Actas del XXII Encuentro de Geohistoria Regional. Pp. 72-76. ISBN 950-692-060-5.

Calandra H. A., Balbarrey G., Couso G., Lamenza G., Aguirre B., Duhalde. N. 2004. El Sitio Las Bolivianas (Formosa): análisis comparativo del material cerámico del Sector Central del Gran Chaco argentino. En actas XXIV Encuentro de Geohistoria Regional (IIGHI-CONICET). Publicado en CDrom formato libro.

Calandra H. A., Santini M., Salceda S., Lamenza G. 2004. Arqueología ribereña del Chaco: Presentación de un nuevo sitio arqueológico". XXIV Encuentro de Geohistoria Regional (IIGHICONICET). Publicado en CDrom formato libro.

Calandra H.A., Salceda S.A., 2004. El territorio y sus ocupantes: ¿qué, quienes, cómo y cuándo?. FOLIA Histórica del Nordeste N 15: 107-127. ISSN 0325-8238.

Colazo S., Méndez M.G., Calandra H.A., Ferrarini S.O., Salceda S.A., 2001. Estudio preliminar del sitio arqueológico "El Chancho", Departamento de San Fernando. Provincia del Chaco. En Actas del XXI Encuentro de Geohistoria Regional. Pp 68-74.

Colazo S., Méndez M.G., Salceda S.A., 2004. El aporte de las fuentes documentales, arqueológicas y antropológicas para el conocimiento de la zona de Colonia Tacuarí, Chaco Austral. Revista Nordeste - Investigación y Ensayos - 2da. Época, № 23: 67-80. ISSN 0328-5995.

Colazo S., Guarino G., 2005. El sitio del Km. 75. Una revisión de sus investigaciones. En Actas del XXV Encuentro de Geohistoria Regional. IIGHI-CONICET. Corrientes. Publicado en CDrom formato libro.

De Feo, C., H.A. Calandra., M. Santini., B. Aguirre., G. Lamenza., M.I. Lanciotti., L. Del Papa \& A. Porterie. 2002. Localización espacial y caracterización cultural de sitios arqueológicos del Gran Chaco Meridional. En: XXII Encuentro de Geohistoria Regional. 263-294 pp. Chaco, Argentina.

Desántolo B., Santini M., Salceda S., 2005. Arqueologia chaqueña 4: Hallazgo de restos oseos humanos en el sitio arqueológico "El Cachape Potrero V" (Prov. de Chaco): Informe preliminar. En Actas del XXV Encuentro de Geohistoria Regional. IIGHI-CONICET. Corrientes. Publicado en CDrom formato libro.

Dougherty B., Zagaglia E.L., 1982. Problemas generales de la arqueología del Chaco occidental. Revista del Museo de La Plata, Nueva Serie, Tomo VIII, Sección Antropología N54: 107-110.

Dougherty B., C. De Feo y H. Calandra. 1992. Arqueología de Formosa. En: Hacia una Nueva Carta Étnica del Gran Chaco, pp 119-125. Centro del Hombre Antiguo Chaqueño (CHACO) IV. Las Lomitas, Pcia. de Formosa, Argentina.

Espiago J., y Baena J. 1999. Los Sistemas de Información Geográfica como tecnología informática aplicada a la Arqueología y a la gestión del patrimonio. En: Los SIG y el análisis espacial en arqueología. Baena, Blasco y Quesada eds. Colección de Estudios. UAM Ediciones.

Lamenza G., Aguirre B., Calandra H. 2005. Alfarería arqueológica del Sector Paraguay Paraná del Chaco Meridional: su sistematización e identidad. En Actas del XXV Encuentro de Geohistoria Regional. IIGHI-CONICET. Corrientes. Publicado en CDrom formato libro. 
Méndez M.G., Calandra H.A., Ferrarini S.O., Salceda S.A., 2000. De la prehistoria a la historia en el Gran Chaco Argentino. Unidad y Diversidad en América Latina. Conflictos y Coincidencias. Tomo 1, Cap. 9: 123-143. María C. Longinotti (Ed). ISBN 987-96037-4-5.

Méndez M.G., Calandra H.A., Ferrarini S.O., Salceda S.A., Tobisch A.C., 2003. Arqueología Chaqueña 2: Nota preliminar sobre un hallazgo de restos óseos humanos en urna. En Actas del XIII Congreso Nacional de Arqueología Argentina. Ed. Brujas. Tomo 3: 91-103.

Naumann M. 1999. Pequeño Atlas Argentino con el Gran Chaco. Programa de acción nacional de lucha contra la destrucción. PAN. Convenio SRNyDS-INTA-GTZ.

Nordenskiöld Erland. 1903. Präcolumbische Wohn-und Begrabnisplatze an der Süd-Westgrenze von Chaco. Kungl. Suenska Vetenskaps Akad. Handlingär, 36 (7). Stockholm.

Primera Convención Nacional de Antropología (Segunda Parte). Normas Mínimas generales para la descripción de yacimientos arqueológicos (1965). Facultad de Humanidades. Universidad Nacional del Nordeste.

Salceda S.A., Méndez M.G., 1999. La biodiversidad amerindia. Tomo 1: La argentina aborigen. Conquista y colonización. Capítulo 2: 65-81. Nueva Historia de la Nación Argentina. Academia Nacional de la Historia. Editorial Planeta. ISBN 950-49-0215-4.

Salceda S.A.; M.G. Méndez; H.A. Calandra; M. Santini; M.A. Giovanetti; G. Couso. 2000. Análisis preliminar de los restos faunísticos del sitio arqueológico "El Cachapé" (Chaco, Argentina). En: XX Encuentro de Geohistoria Regional. Vol II: 795-806. Resistencia, Chaco.

Salceda S.A., Calandra H.A., 2003. La planicie chaqueña: orígenes de su historia. Academia Nacional de la Historia. Buenos Aires. Pp. 1-18.

Santini M., Salceda S.A., De Santis L., Del Papa L., 2003. Primeras aproximaciones a estudios tafonómicos en sitios de la región ribereña-paranaense del Chaco meridional. En Actas del XXIII Encuentro de Geohistoria Regional. Pp. 364-369.

Sempé M.C., Calandra H.A., Salceda S.A., Rizzo A., De Feo C. 2003. Mesopotamia argentina: arqueología del alto río Uruguay. En Actas del XXIII Encuentro de Geohistoria Regional. Pp. 242-251.

Renfrew C., Bahn P., 2000. ARCHAEOLOGY: Theories Methods and Practice. UK, Thames \& Hudson.

Rosen E. von. 1957 (1917), Un mundo que se va. Exploraciones y aventuras entre las altas cumbres de la Cordillera de los Andes. Opera Lilloana $N^{\circ} 1$. Fundación Miguel Lillo, Instituto Miguel Lillo, Universidad Nacional de Tucumán, 307 págs.

Tarragó, M. y V. Núñez Regueiro. 1972. Un diseño de Investigación Arqueológica para el Valle Calchaquí. Fase exploratoria. Estudios de Arqueología N 1: 62-85 apénd. 3:78 Museo Arqueológico de Cachi. Cachi, Salta.

Tomasini, A.; Braunstein, J.; Calandra, H.A. 2003. Síntesis histórica y localización de la primera ciudad de Esteco (1566-1609). En: La mitad verde del Mundo Andino. Estado actual de las investigaciones arqueológicas en la vertiente oriental de los Andes y las tierras bajas de Bolivia y Argentina Ventura, B.N. y Ortiz G. (Eds). Pp. 285-316. 